\title{
Group B streptococcal infection
}

\author{
A review and update
}

JOHN M. KNOX

From the Department of Dermatology, Baylor College of Medicine, Houston, Texas, USA

SUMMARY The problems posed by the sudden increase in serious group B streptococcal disease among infants since the early 1970 s are described and discussed. Virtually all offspring of colonised mothers harbour the organism and infection develops in about $1 \%$ of these infants. The mortality rate, even with immediate antibiotic treatment of early onset disease, is $30 \%$; delay in treatment leads to much higher mortality. Late onset disease starting around the seventh to ninth day of life, but sometimes as late as the second month, is less frequently fatal. Preventive measures include active and passive immunisation or intravenous ampicillin during labour. Experimental evidence indicates that each of these methods gives protection.

\section{Introduction}

Sexual activity of the parents may be responsible for the emergence of many cases of serious $\beta$-haemolytic group B streptococcal infections among newborn infants. Although $\beta$-haemolytic group B streptococcus has been identified as a cause of puerperal and neonatal disease since 1938, the incidence of infection remained low until the late 1960s and early 1970s, when a dramatic increase in serious group B streptococcal infection among infants was noted. Currently, it is predicted that between 12000 and 15000 infants in the United States will develop group B streptococcal disease during the next year (Horn et al., 1974). In a number of geographical areas, the group B streptococcus is now the leading cause of meningitis during the first two months of life (Franciosi et al., 1973; Baker et al., 1973). Approximately $50 \%$ of affected infants will die; approximately $50 \%$ of the survivors with meningeal invasion will develop neurological sequelae (Horn et al., 1974). Because the incidence of serious infection caused by other bacteria has not diminished in recent years, the increase of group B streptococcal disease has resulted in an absolute increase in the number of

Address for reprints: Professor John M. Knox, Department of Dermatology, Baylor College of Medicine, Texas Medical Center, Houston, Texas 77030, USA

Received for publication 21 November 1978 cases of serious neonatal bacterial infections in our city (McCracken, 1976).

The reason for the emergence of the group $B$ streptococcus as a cause of serious neonatal infections is unknown. This has prompted numerous investigations concerning colonisation of pregnant women (Franciosi et al., 1973; Baker and Barrett, 1973; Bevanger, 1974; Schauf and Hlaing, 1976; Ferrieri et al., 1977; Aber et al., 1976). Group B streptococci can often be found on normal human mucous membranes. Selective media cultures indicate that from $25 \%$ to $35 \%$ of asymptomatic pregnant women carry group B streptococci in their vagina. Pharyngeal and anal colonisation are also not uncommon. In approximately $50 \%$ of the cases studied, male consorts of colonised women will show recovery of the same serotype streptococcus from their urethra (Eichenwald, 1978).

\section{EARLY ONSET DISEASE}

Early onset disease in infants is caused by the same serotype as that which colonises the mother's vaginal tract. This finding indicates that the baby becomes infected either in utero or during the birth process. Up to $70 \%$ of vertical transmission of group B streptococci from mother to infant, presumably at the time of delivery, has been reported (Baker and Barrett, 1973; Aber et al., 1976). Virtually all offspring of colonised mothers harbour the organisms in one or several sites. Infection develops in about $1 \%$ of these infants. Neonatal streptococcal illness may be caused by all 
of the five known serotypes (1A, 1B, 1C, type 2 and type 3) (Eichenwald, 1978).

Early onset streptococcal disease is a critical illness, usually appearing during the first day of life, although symptoms may be delayed for as long as one week. The syndrome is characterised by acute respiratory distress, apnoea, and shock often associated with septicaemia and meningitis., The illness closely resembles hyaline membrane disease, with the lung being the initial and major site of involvement, presumably infected by aspiration of infected vaginal secretions or amniotic fluid. Cultures of the nasopharynx, gastric aspirate, skin, and meconium usually give positive results, as do blood cultures. The ratio of immature to mature polymorphonuclear leucocytes in the peripheral blood may aid in distinguishing group B streptococcal infection from hyaline membrane disease.

Early onset disease is extremely serious; although antimicrobial agents are available for the treatment of group B streptococcal infection, even promptly administered antibiotics are often unable to prevent fatal progression, mortality rates being approximately $30 \%$ (Eichenwald, 1978; National Institutes of Health, 1972). When therapy is delayed until laboratory confirmation of group B streptococci is received, most infected infants will die. In our teaching hospitals newborn infants with respiratory distress are treated immediately with antibiotics; these medications are continued until blood cultures have failed to grow the pathogen.

\section{LATE ONSET DISEASE}

Late onset group B streptococcal disease is a more limited and less severe type of infection. Symptoms usually begin around the seventh to ninth day of life but may appear as late as the second month. Late onset disease is usually a focal infection and may produce such diverse syndromes as meningitis, cellulitis, arthritis, osteomyelitis, otitis media, ethmoiditis, conjunctivitis, renal infections, purulent pericarditis, and brain abscess (Eichenwald, 1978). Mortality from late onset illness is considerably lower than from early onset disease; however, there is a high incidence of permanent residua. Almost one half of infants presenting with late onset disease are born from non-colonised mothers; furthermore, there is little correlation between the serotype recovered from the infants and that cultured from colonised mothers. These findings indicate that late onset infection is probably acquired by a different mechanism than is early onset disease. Rather than being an expression of a longer incubation period after intrapartum infection, late onset disease is probably acquired from the nursery or at home.

\section{Research at Baylor College of Medicine}

Maternal colonisation surveillance continues to be of interest. My associates recently described the prevalence of group B streptococcal colonisation among lower socioeconomic parturients, ascertaining demographic data regarding age, race, marital status, parity, and trimester of pregnancy for 112 women (Beachler et al., 1979). At the time of enrolment in the study, single vaginal and throat swabs were obtained and immediately inoculated into a selective broth medium containing ToddHewitt broth (Difco Laboratories), 5\% defibrinated sheep red blood cells, gentamicin sulphate $8 \mu \mathrm{g} / \mathrm{ml}$ (Schering Corp.), and nalidixic acid 15 $\mu \mathrm{g} / \mathrm{ml}$ (Winthrop Laboratories) (Beachler et al., 1979). The broth cultures were incubated overnight at $35^{\circ} \mathrm{C}$ and streaked on to sheep blood agar plates, with colonies of non- $\beta$-haemolytic and $\beta$-haemolytic streptococci being subsequently serogrouped and serotyped by methods described previously (Franciosi, 1973).

\section{Results}

Group B streptococci were recovered from 32 $(28.6 \%)$ of the study participants. Colonisation at vaginal sites alone occurred in 26 subjects $(23.4 \%)$ and at throat sites alone in five $(4.4 \%)$. In one patient, streptococci of identical serotype were recovered from both throat and vagina. Thirtythree strains of group B streptococci were isolated from throat and vaginal cultures, with type 3 being the most frequent $(39.5 \%)$; type $2(30 \cdot 3 \%)$, 1B $(21 \cdot 2 \%), 1 \mathrm{~A}(3.0 \%)$, and $1 \mathrm{C}(3.0 \%)$ were isolated less frequently. No statistically significant differences were detected between colonised and noncolonised pregnant women with regard to age, race, marital state, or parity.

Ninety-six of the study participants had sera available for detection of antibody directed against the type 3 capsular polysaccharide antigen. Sera were collected at the time of enrolment in the study and stored at $-20^{\circ} \mathrm{C}$ until tested. Serum concentration of antibody was determined by radioactive antigen-binding assay (RABA) (Baker and Kasper, 1976; Baker et al., 1977). Sera from 65 non-colonised control subjects showed a median antibody concentration of $1.00 \mu \mathrm{g} / \mathrm{ml}$ (range $0 \cdot 376->40 \cdot 3 \mu \mathrm{g} / \mathrm{ml}$ ). Thirty-one women colonised with any of the group B streptococcal serotypes showed a median antibody concentration of $1 \cdot 12$ $\mu \mathrm{g} / \mathrm{ml}$ (range $0.442->40.3 \mu \mathrm{g} / \mathrm{ml}$ ), and 13 women colonised with type 3 streptococci had a median antibody concentration of $4.84 \mu \mathrm{g} / \mathrm{ml}$ (range $0 \cdot 550->40 \cdot 3 \mu \mathrm{g} / \mathrm{ml}$ ). Concentration of antibody 
to the type 3 capsular polysaccharide in sera from non-colonised subjects was significantly lower than that in sera from women colonised with type 3 streptococci $(P=0.0241)$. No statistically significant differences, however, were found in sera from women colonised with the various serotypes of group B streptococci $(P=0.0863$, Mann-Whitney $U$ test) (Snedecor and Cochran, 1967). Frequency distribution of concentration of serum antibody in these 96 parturients studied was similar to that previously reported from pregnant women, neonates, infants, and children; the level of serum antibody may be related in part to increasing age among infants and children (Baker and Kasper, 1976; Baker et al., 1977; Hammerschlag et al., 1977).

\section{Discussion}

Little is known concerning the relationship between asymptomatic mucous membrane colonisation with the group B streptococcus in the pregnant woman and the presence of serum antibody to the surface antigens of this bacterium. Recently, Baker and Kasper (1976) demonstrated a significant correlation between very low levels of antibody to the capsular polysaccharide of type 3 group B streptococci in women harbouring type 3 organisms genitally and risk for invasive neonatal disease due to these strains (Baker and Kasper, 1976). Their studies also indicated that low levels of type 3-specific antibody can be detected in sera from women of all ages (Baker et al., 1977; Hammerschlag et al., 1977; Hemming et al., 1976).

Three methods for the prevention of infant colonisation with group B streptococci have been suggested: (1) eradication of vaginal colonisation (and perhaps rectal colonisation) of the pregnant woman by treating her and her spouse with oral penicillin; (2) temporary eradication of vaginal group B streptococci carriage during the intrapartum period by administration of an intravenous penicillin during labour; and (3) penicillin treatment of the infant at the time of birth. The first two of these methods are being investigated by our group. To date, the couple-treatment investigation has yielded disappointing results, as have similar studies by others (Hall et al., 1976). Results of the intravenous antibiotic treatment of women during labour are not complete but appear promising.

If it is correct that antibody-deficient infants are more likely to develop early or late onset disease, two possible immunological approaches are suggested: (1) administration of specific high titre immune globulin to all infants immediately after birth, which could theoretically prevent late onset disease and perhaps reduce the incidence of early onset disease as well; and (2) development of a polyvalent antistreptococcal vaccine to be administered during pregnancy. Observations in mice with one serotype indicate a considerable degree of protection with each of the above approaches (Eichenwald, 1978). The present lack of any alternative method of prevention, except intravenous ampicillin administered to all mothers harbouring the organism, indicates the need for study of possible methods of immune prophylaxis.

\section{References}

Aber, R. C., Allen, N., Howell, J. T., Wilkinson, H. W., and Facklam, R. R. (1976). Nosocomial transmission of group B streptococci. Pediatrics, 58, 346.

Baker, C. J., and Barrett, F. F. (1973). Transmission of group B streptococci among parturient women and their neonates. Journal of Pediatrics, 83, 919.

Baker, C. J., Barrett, F. F., Gordon, R. C., and Yow, M. D. (1973). Suppurative meningitis due to streptococci of Lancefield group B: a study in 33 infants. Journal of Pediatrics, 82, 724.

Baker, C. J., and Kasper, D. L. (1976). Correlation of maternal antibody deficiency with susceptibility to neonatal group B streptococcal infection. New England Journal of Medicine, 294, 753.

Baker, C. J., Kasper, D. L., Tager, I. B., Paredes, A., Alpert, S., McCormack, W. M., and Goroff, D. (1977). Quantitative determination of antibody to capsular polysaccharide in infection with type III strains of group B streptococcus. Journal of Clinical Investigation, 59, 810.

Beachler, C. W., Baker, C. J., Kasper, D. L., Fleming, D. K., Webb, B. J., and Yow, M. D. (1979). Group B streptococcal colonization and antibody status in lower socioeconomic parturient women. American Journal of Obstetrics and Gynecology (in press).

Bevanger, L. (1974). Carrier rate of group B streptococci with relevance to neonatal infections. Infection, $2,123$.

Eichenwald, J. F. (1978). Group B streptococcal disease of infancy. Infectious Diseases, 8, (3), 4.

Ferrieri, P., Cleary, P. P., and Seeds, A. E. (1977). Epidemiology of group B streptococcal carriage in pregnant women and newborn infants. Journal of Medical Microbiology, 10, 103.

Franciosi, R. A., Knostman, J. D., and Zimmerman, R. A. (1973). Group B streptococcal neonatal and infant infections. Journal of Pediatrics, 82, 707.

Hall, R. T., Barnes, W., Krishnan, L., Harris, D. J., Rhodes, P. G., Foyez, J., Miller, G. L. (1976). Antibiotic treatment of parturient women colonized with group B streptococci. American Journal of Obstetrics and Gynecology, 124, 630.

Hammerschlag, M. R., Baker, C. J., Alpert, S., Kasper, D. L., Rosner, I., Thurston, P., Webb, B. J., and McCormack, W. M. (1977). Colonization with group $B$ streptococci in girls under 16 years of age. Pediatrics, 60, 473.

Hemming, V. G., Hall, R. T., Rhodes, P. G., Shigeoka, A. O., and Hill, H. R. (1976). Assessment of group B streptococcal opsonins in human and rabbit serum by neutrophil chemiluminescence. Journal of Clinical Investigation, 58, 1379.

Horn, K. A., Zimmerman, R. A., Knostman, J. D., and Meyer, W. T. (1974). Neurological sequelae of group B streptococcal neonatal infections. Pediatrics, 53, 501.

McCracken, G. H., jun. (1976). Editorial comment. Journal of Pediatrics, 89, 203.

National Institutes of Health (1977). Summary of the workshop on perinatal infections due to group B streptococcus. Journal of Infectious Diseases, 137, (1), 137.

Snedecor, G. W., and Cochran, W. G. (1976). Statistical Methods, p. 593. Iowa State University Press: Ames, Iowa. 